\title{
Proinflammatory Cytokines in the Saliva, Gingival Crevicular Fluid and Serum of Diabetic Patients with Periodontal Disease
}

\author{
Fawad Javed $^{1}$ and Asma Ahmed ${ }^{2}$ \\ ${ }^{1}$ Research Chair for Growth Factors and Bone Regeneration, 3D Imaging and Biomechanical \\ Laboratory, College of Applied Medical Sciences, King Saud University, Riyadh, Saudi Arabia \\ ${ }^{2}$ Aachen dental Laser Centre, RWTH Aachen University, Aachen, Germany
}

Correspondence should be addressed to: Fawad Javed; fawjav@gmail.com

Received 20 May 2013; Accepted 23 June 2013; Published 29 August 2013

Academic Editor: Nurcan Buduneli

Copyright (C) 2013 Fawad Javed and Asma Ahmed. Distributed under Creative Commons CC-BY 3.0

\begin{abstract}
Periodontal disease (PD) is a complication of diabetes mellitus (or diabetes). Various proinflammatory cytokines are expressed in the saliva, gingival crevicular fluid (GCF) and serum of diabetic patients with PD. The aim of this study was to review the proinflammatory cytokinees that are expressed in the saliva, GCF and serum of diabetic patients with PD. The addressed focused question was "Which proinflammatory cytokines are expressed in the saliva, GCF and serum of diabetic patients with PD?" Databases were explored from 1991 up to and including May 2013 using various combinations of keywords. Articles published only in English were included. Historic reviews, unpublished data, and review articles were excluded. Levels of interleukin (IL)- 6 are higher in the saliva, serum and GCF of diabetic patients with PD compared to healthy controls. IL-1 $\beta$ and tumor necrosis factor-alpha (TNF- $\alpha$ ) are higher in the serum and GCF of diabetic patients in PD compared to healthy controls. Weak evidence also GCF and serum suggested that resistin and visfatin are also dysregulated in diabetic patients with PD compared to controls. Raised levels of proinflammatory cytokines (including IL-1 $\beta$, IL- 6 and TNF- $\alpha$ ) in the saliva, serum and GCF of diabetic patients with PD may be a valuable tool in the early detection of PD. Elevated concentrations of proinflammatory cytokines in the saliva, serum and/or GCF may also be indicative of "latent" diabetes in undiagnosed individuals.
\end{abstract}

Keywords: Cytokine, periodontal disease, saliva, serum, gingival crevicular fluid, diabetes mellitus.

\section{Introduction}

Periodontal disease (PD) jeopardizes the supporting structures of teeth including periodontal ligament and alveolar bone). PD is caused by an imbalance between periodontal pathogens and host responses
(Costa et al., 2010). If left untreated, PD may cause destruction of these supporting structures and ultimately cause tooth loss (Costa et al. 2010; Cutando et al., 2003; Gomes et al., 2006). Significant risk factors for PD are chronic hyperglycemic conditions such as poorly-controlled type 2 
diabetes mellitus (T2DM) and prediabetes (Bandyopadhyay et al., 2010; Javed et al, 2013a; Javed et al., 2009; Javed et al., 2007; Javed and Romanos 2009; Javed et al., 2013b). Persistent hyperglycemia modulates periodontal tissue destruction by altering the function of polymorphonuclear (PMN) leukocytes, collagen and glycosaminoglycan synthesis, formation of advanced glycation end products (AGEs), and deregulating cytokine production (Chen et al., 2010; Dag et al., 2009; Kardesler et al., 2010; Lalla et al., 2007; O'Connell et al, 2008).

Cytokines are water-soluble glycoproteins secreted by hematopoietic and nonhematopoietic cells in response to infection. Their primary function is intercellular signaling (Holloway et al, 2002). An inflammatory cytokine may be described as a cytokine which is induced during an inflammatory response and is associated with the onset and/or progression of the insult. So far, interleukin (IL)-l alpha ( $\alpha$ ) ILbeta $(\beta)$ IL-6, IL-8, and tumor necrosis factor (TNF)- alpha $(\alpha)$ have been categorized as inflammatory cytokines. Since alveolar bone resorption is a classical feature of PD, studies have paid particular attention to the roles of inflammatory cytokines in saliva (Costa et al., 2010; Cutando et al., 2003; Gomes et al., 2006), gingival crevicular fluid (GCF) (Cole et al., 2008; Correa et al., 2008; Engebretson et al., 2006; Fitzsimmons et al., 2010; Kardesler et al, 2008; Nareika et al., 2007; NavarroSanchez et al., 2007; Salvi et al., 1998; Salvi et al., 2010; Salvi et al., 1997b) and serum (Chen et al, 2010; Dag et al, 2009; Engebretson et al., 2007; Ioannidou et al, 2006; Kardesler et al., 2010; Lalla et al., 2006; Nishimura et al., 2006; O'Connell et al, 2008; Pradeep et al., 2010) of individuals with PD. Simultaneously, the increased prevalence of PD in patients with poorlycontrolled diabetes may be explained by the role of TNF- $\alpha$ which plays a predominant role in inducing insulin resistance in obese subjects (Nishimura and Murayama 2001). It has been hypothesized that TNF- $\alpha$ levels in the circulation of diabetic subjects are influenced by periodontal infection and inflammation (Nishimura and Murayama
2001). Therefore, periodontal treatment may improve the metabolic control of diabetes via improved insulin sensitivity by reducing the peripheral TNF- $\alpha$ concentration.

Although separate studies have investigated the cytokine expressions in saliva, GCF and serum in diabetic patients with $\mathrm{PD}$; we observed that a review article that would provide an overview of the potent proinflammatory cytokines expressed in the saliva, GCF and serum of diabetic patients with PD is not yet available. In this context, the aim of the present study was to comprehensively review the potent proinflammatory cytokines in saliva, gingival crevicular fluid and serum of diabetic patients with PD.

\section{Materials and methods}

\section{Focused Question}

The addressed focused question was "Which proinflammatory cytokines are expressed in the saliva, GCF and serum of diabetic patients with PD?"

\section{Search Strategy}

As a first step, the authors (FJ and HBA) searched the MEDLINE/PubMed (National Library of Medicine, Bethesda, Maryland, USA) and Google-Scholar databases using the following keywords in various combinations: "cytokine", "gingival crevicular fluid", "saliva", "serum" and "plasma". Databases were explored from 1991 up to and including May 2013. Titles and abstracts of articles that satisfied the selection protocol were screened by the authors and checked for agreement. The full-text of the articles judged by title and abstract to be relevant were read and independently assessed against the selection protocol. This was followed by hand-searching of the reference lists of original and review studies that were found to be relevant in the previous step and once again, any disagreement between the authors was resolved via discussion (Figure 1). 
Which proinflammatory cytokines are expressed in the saliva, gingival crevicular fluid and serum of diabetic patients with periodontal disease?

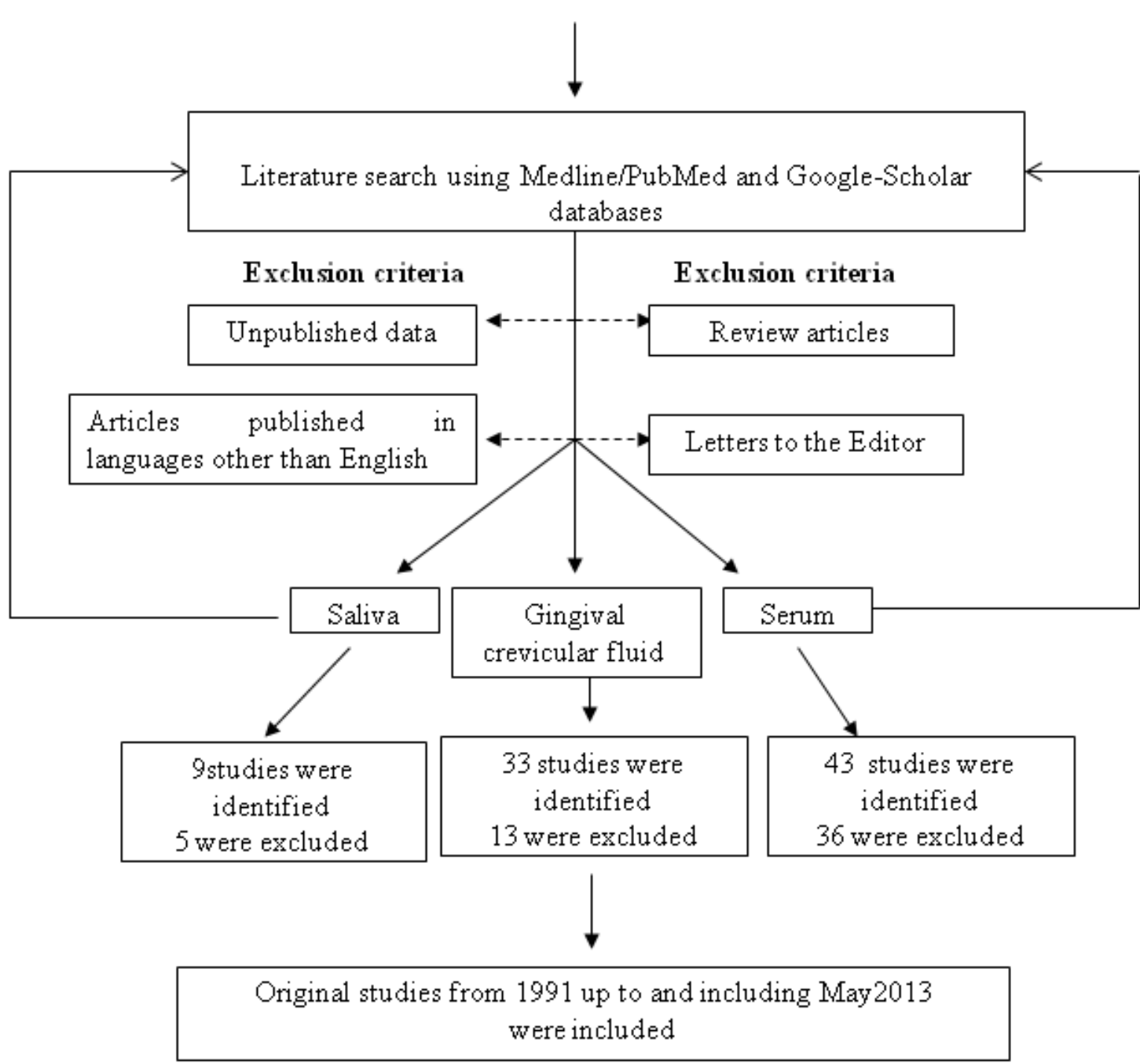

Figure 1: A Diagrammatic Representation of the Study Selection Protocol

\section{Study Selection Criteria}

The study selection criteria encompassed the following: 1) Original articles; 2) human studies; 3) Reference list of original and review studies; 4) use of statistical methods; 5) Intervention: cytokine profile in saliva, serum and GCF in diabetic patients with PD; and 6) Articles published only in Englishlanguage.
The studies that were included in the present review were controlled for confounding parameters including age, smoking, body mass index, medication and alcohol consumption. Letters to the editor, historic reviews and unpublished articles were excluded. 


\section{Cytokine Profiles in Diabetic Patients with Periodontal Disease: Lessons Learned from Previous Studies}

\section{Unstimulated Whole Saliva}

Costa et al., (2010) investigated the concentrations of IL- 6 in unstimulated whole saliva (UWS) samples collected from type 2 diabetic patients with PD (age range 35-65 years). In this study (Costa et al, $2010)$, the diabetic participants $(n=44)$ had T2DM since at least 5 years and had a poor metabolic control over the metabolic disorder (Hemoglobin A1C (HbA1c) > 6\%). The IL- 6 concentrations in the saliva of PD patients with or without T2DM were significantly higher compared to those in healthy controls. These results are in accordance with the function of IL- 6 on the modulation of periodontal inflammation (Bartold and Haynes 1991). IL-6 dictates the transition from acute to chronic inflammation by changing the nature of leukocyte infiltrate (from PMN neutrophils to monocytes/macrophages) exerts stimulatory effects on $\mathrm{T}$ and $\mathrm{B}$ cells, and induces antibody formation, favoring a chronic inflammatory response (Bartold and Haynes 1991).

Gomes et al., (2006) investigated the levels of interferon-gamma (IFN- $\gamma$ ) and TNF- $\alpha$ with PD in patients with T2DM. In this study, nearly $63 \%$ of the participants had well-controlled T2DM (Gomes et al., 2006). The results showed increased levels of IFN- $\gamma$ in $60 \%$ and approximately $15 \%$ of the patients with severe- and moderate PD respectively; however, there were no significant differences between TNF- $\alpha$ levels between the groups (Gomes et al, 2006). Immunoregulatory properties of IFN $-\gamma$ may act as a possible explanation for the insignificant differences in TNF- $\alpha$ levels between diabetic subjects with moderate and severe PD (Gemmell and Seymour 1994). It should however be remembered that UWS is a mixture of molecules derived from the major and minor salivary glands, the mucosal epithelium, as well as the GCF. Therefore, it may be argued that the expression of proinflammatory cytokines in UWS may in fact be due to the leakage of GCF into the oral cavity of diabetic patients with PD. In addition, salivary cytokine levels in UWS affected mainly by the amount of blood contamination (which is a common manifestation in patients with PD) (Suh et al, 2009). Further studies are necessary to fully explore the potential impacts of diabetes and PD on the expression of proinflammatory cytokines in UWS.

\section{Gingival Crevicular Fluid}

GCF is a serum transudate which exists in the gingival sulcus (as an inflammatory exudate) of patients with PD. Since GCF collection is a non-invasive technique, there is growing interest for its use in monitoring periodontal inflammation. In their study, Fitzsimmons et al., (2010) investigated the independent and combined associations between GCF IL-1 $\beta$ and PD. In this study, GCF samples were collected from 939 individuals and IL-1 $\beta$ levels were investigated using Enzyme linked immunosorbent assay (ELISA) (Fitzsimmons et al., 2010). The results showed raised levels of IL- $1 \beta$ in diabetic patients with PD compared to non-diabetic controls (Esposito et al., 2002). Likewise, another study compared the levels of biomarkers in the GCF between patients with type 1 diabetes and healthy subjects during experimental gingivitis (Salvi et al, 2010). In this study, GCF biomarkers were compared between nine, type 1 diabetesic and a similar number of control individuals (Salvi et al. 2010). There was no significant difference in age and gender between the groups. The results showed that the mean GCF biomarker levels for IL-1 $\beta$ and matrix metalloproteinase (MMP)-8 were significantly higher in patients with type 1 diabetes compared to healthy non-diabetic individuals (Salvi et al, 2010). Since IL-1 $\beta$ is a proinflammatory and bone-resorbing cytokine that has been positively associated with the progression of PD; it may be postulated that early treatment of periodontal inflammatory conditions may help reduce the levels of this cytokine in the GCF. This may in turn, retard the periodontal inflammatory conditions in susceptible individuals, such as those with diabetes. Correa et al., (2008) investigated the effectiveness of non-surgical periodontal therapy in improving the periodontal status 
and reducing the levels of IL-1 $\beta$, IL-18, MMP-8 and -9 levels in the GCF among periodontitis patients with and without T2DM. The results showed that nonsurgical periodontal therapy significantly reduced the clinical parameters of periodontal inflammation (including gingival bleeding and probing pocket depth). This was accompanied by a significant reduction in IL-1 $\beta$, MMP-8 and 9 levels in deep sites (Correa et al., 2008). However, in the study by Kardeşler et al., (2008) T2DM did not influence the expression of proinflammatory mediators in the GCF. Similarly, results by Engebretson et al., (2006) also showed that GCF levels of IL-8 are not associated with the severity of T2DM. Further studies are warranted in this regard.

\section{Serum}

Increased circulating levels of IL- 6 have been associated with the progression of diabetes (Esposito et al, 2002). Nonsurgical periodontal therapy has been reported to significantly reduce the serum concentrations of IL- 6 thereby reducing the elevated glycemic levels as well as periodontal inflammation. The study by O'Connell et al., (2008) investigated the effect of non-surgical periodontal treatment on the levels of circulating proinflammatory cytokines in diabetic individuals with PD. The results showed a significant reduction in the serum concentrations of IL- 6 and IL12 following periodontal therapy in patients with T2DM (O'Connell et al., 2008).

TNF- $\alpha$ has been reported to play a significant role in the pathogenesis of T2DM by inhibiting insulin action (Argiles et al., 1994; Salvi et al, 1997a). Results by Dağ et al., (11) showed a significant reduction in the serum concentrations of TNF- $\alpha$ in type 2 diabetic patients with PD following non-surgical periodontal treatment. A dose-response relationship between PD severity and serum levels of TNF- $\alpha$ has also been reported (Salvi et al., 1997a). Results by Salvi et al, (1997a) suggested that the diabetic state results in an upregulated monocytic TNF- $\alpha$ secretion which, in the presence of gram-negative bacteria, intensifies the expression of PD.

\section{Expression of Other Cytokines in the Saliva, Serum and Gingival Crevicular Fluid of Diabetic Patients with Periodontal Disease}

Visfatin (initially termed, human pre-B-cell colony enhancing factor) is a 52 kilodalton molecule, secreted by adipocytes. It has been suggested that visfatin induces the production of IL-1 $\beta$, TNF- $\alpha$ and IL- 6 during infection and inflammation. To our knowledge from indexed literature, only one study has assessed visfatin levels in the GCF and serum of type 2 diabetic patients with periodontal disease (Pradeep et al., 2012). In this study, 30 patients were divided into three groups: group 1 (10 healthy), group 2 (10 patients with well-controlled T2DM and PD), and group 3 (10 individuals with CP and without diabetes). Visfatin levels in the serum and GCF were determined using ELISA. The results demonstrated that the mean visfatin concentration was higher in serum and GCF in individuals with T2DM with PD compared to healthy controls (Pradeep et al., 2012). Furthermore, the results also showed that serum and GCF visfatin levels correlated positively with the clinical periodontal inflammatory parameters (Pradeep et al, 2012). It is tempting to speculate that visfatin are also higher in diabetic patients with $\mathrm{PD}$ as compared to systemically healthy control; however, further studies are warranted to assess this relationship.

Resistin, an adipocytokine that induces insulin resistance, is majorly expressed in adipocytes and peripheral blood mononuclear cells. Expression of resistin is increased in inflammatory diseases as well as diabetes mellitus, and is upregulated by bacterial pathogens and proinflammatory cytokines (Hiroshima et al., 2012). In indexed literature, only one study could be cited which aimed to identify resistin in human GCF among patients with and without periodontitis and diabetes (Hiroshima et al., 2012). In this study, GCF samples were collected from 24 patients with PD, 18 patients with diabetes mellitusrelated PD and 21 healthy controls. Resistin in gingival crevicular fluid was determined using western blot analysis and ELISA. The results demonstrated that the resistin level 
in the GCF from patients with PD or diabetes mellitus-related PD were significantly higher than those identified in healthy controls (Hiroshima et al., 2012). It is hypothesized that resistin levels in the saliva and/or serum of patients with OD and diabetes; however, this hypothesis is yet to be confirmed via future investigations.

\section{Discussion}

In the present comprehensive literature review, we aimed to summarize the proinflammatory cytokines that are expressed in the saliva, serum and GCF of diabetic patients with PD. From the literature reviewed, we observed that there is a dysregulation in cytokine production in diabetic patients with PD as compared to healthy controls (Figure 2).

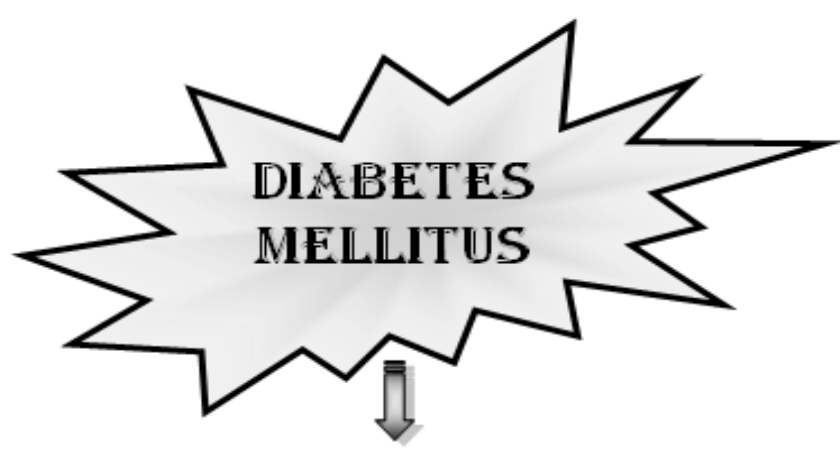

Hyperglycemia

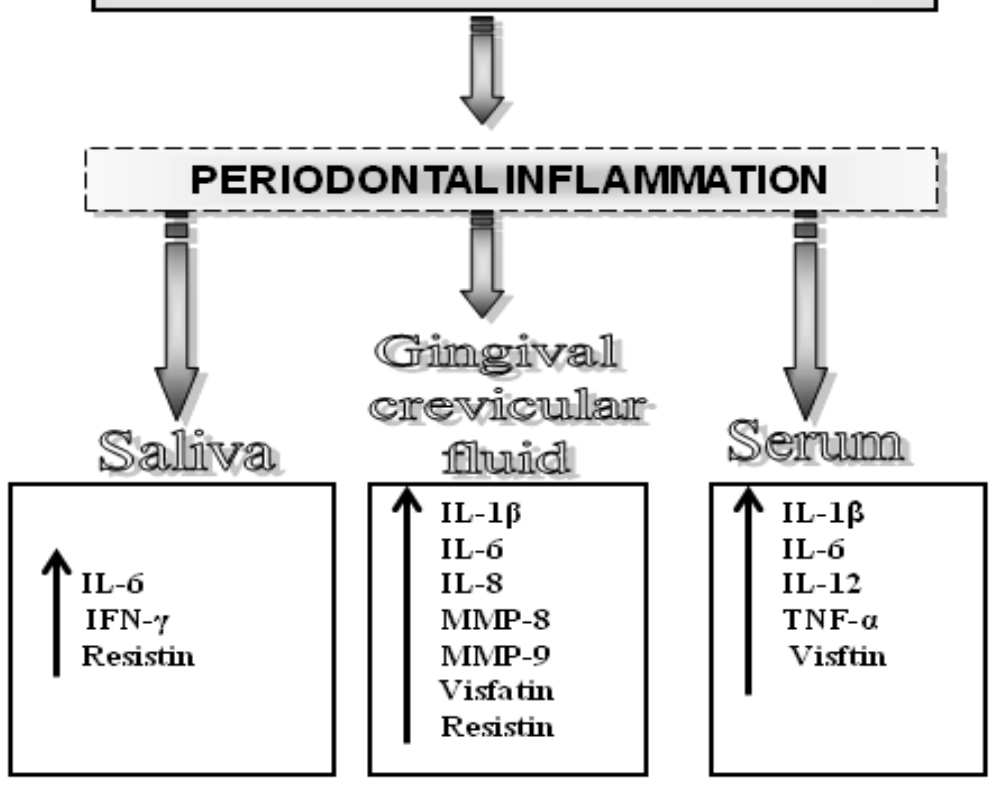

Figure 2: A Diagrammatic Representation of the Cytokine Profile in the Saliva, Gingival Crevicular Fluid and Serum of Diabetic Patients with Periodontal Disease

IL: Interleukin IFN- $\gamma$ : Interferon-gamma MMP: Matrix metalloproteinase

TNF- $\alpha$ : Tumor necrosis factor-alpha

Predominantly, connective tissue destruction that takes place in patients with poorly-controlled diabetes and PD results from the interaction between bacteria (and their products) and PMN cells (Kinane and Bartold 2007; Mealey and Ocampo 2007). In addition, it has been proposed that patients with poorly-controlled diabetes have more 
severe PD due to the increased production and accumulation of glucose mediated AGEs in the periodontal tissues as compared to patients with well-controlled diabetes and healthy controls (Grossi and Genco 1998; Javed et al, 2007). Chronic hyperglycemia affects the migration and phagocytic activity of mononuclear and PMN phagocytic cells resulting in establishment of more pathogenic sub-gingival flora. This triggers an infection-mediated pathway and dysregulates cytokine production and expression. Furthermore, PD amplifies the magnitude of the macrophage response to AGEs thereby enhancing cytokine production and oxidative stress thus amplifying the classical pathway of diabetic connective tissue degradation, destruction and proliferation. Grossi and Genco (1998) proposed that PD mediated cytokine synthesis and secretion may amplify the magnitude of the AGE mediated cytokine response and vice versa. Proinflammatory cytokines such as IL- 6 and TNF- $\alpha$, promote periodontal inflammation, in part, by stimulating the expression of chemokines such as monocyte chemotactic protein 1 and macrophage inflammatory protein 1 that direct the migration of leukocytes into the vessel walls (Gimbrone et al, 1995).

It is well-known that a direct relationship exists between obesity and T2DM (Mealey and Ocampo 2007). Adipose tissues, particularly visceral fat, have been associated with insulin resistance since they may cause insulin resistance through the release of free fatty acids (FFAs). Adipocytes and macrophages associated with visceral fat also synthesize IL-6 and TNF- $\alpha$ (major cytokines responsible for inducing insulin resistance at the receptor level). From the literature reviewed, we learned that IL- 6 has been identified in the saliva, serum as well as GCF of diabetic patients with PD. It is therefore tempting to speculate that glycemic control reduces periodontal inflammation as well as IL-6 and TNF- $\alpha$ levels in saliva, serum and GCF of patients with diabetes. However, further studies are warranted to verify this hypothesis. IL-18 is a pleiotropic proinflammatory cytokine that plays essential regulatory functions in the innate immune response. Activation of interleukin18 has been found to be involved in the pathogenesis of metabolic syndrome.

IL-1 $\beta$, a classic pyrogen, causes redness in periodontal disease, due in part to the capillary wall permeability that occurs in response to this cytokine. Furthermore, IL$1 \beta$ stimulates collagenase production, which in turn breaks down periodontal connective tissues and leads to the formation of osteoclasts and concomitant alveolar bone loss. Although IL-1 $\beta$ has been identified in the GCF and serum of diabetic patients with $\mathrm{PD}$, further studies are needed to determine the salivary levels of IL-1 $\beta$ in diabetic patients with periodontal disease. Due to a limited number of studies that have assessed the expression of visfatin and resistin in GCF of diabetic patients with PD, authors of the present comprehensive review find it exigent to comment on the significance of these cytokines with reference to diabetes and PD.

On a community level, determination of cytokines (particularly IL-6) in saliva, serum or GCF may be a useful tool in estimating the prevalence of PD. Furthermore, such immunologic assays help also in predicting hyperglycemia (or latent diabetes) in undiagnosed individuals.

\section{Conclusion}

Raised levels of proinflammatory cytokines (including IL-1 $\beta$, IL-6 and TNF) in the saliva, serum and GCF of diabetic patients with PD may be a valuable tool in the early detection of PD. Elevated concentrations of proinflammatory cytokines in the saliva, serum and/or GCF may also be indicative of "latent" diabetes in undiagnosed individuals.

\section{References}

Argiles, J. M., Lopez-Soriano, J. \& LopezSoriano, F. J. (1994). "Cytokines and Diabetes: The Final Step? Involvement of TNF-alpha in Both Type I and II Diabetes Mellitus," Hormone and Metabolic Research , 26(10), 447-9. 
Bandyopadhyay, D., Marlow, N. M., Fernandes, J. K. \& Leite, R. S. (2010). "Periodontal Disease Progression and Glycaemic Control among Gullah African Americans with Type-2 Diabetes," Journal of Clinical Periodontology, 37(6), 501-9.

Bartold, P. M. \& Haynes, D. R. (1991). "Interleukin-6 Production by Human Gingival Fibroblasts," Journal of Periodontal Research, 26(4), 339-45.

Chen, L., Wei, B., Li, J., Liu, F., Xuan, D., Xie, B. \& Zhang, J. (2010). "Association of Periodontal Parameters with Metabolic Level and Systemic Inflammatory Markers in Patients with Type 2 Diabetes," Journal of Periodontology, 81(3), 364-71.

Cole, C. M., Sundararaj, K. P., Leite, R. S., Nareika, A., Slate, E. H., Sanders, J. J., LopesVirella, M. F. \& Huang, Y. (2008). "A Trend of Increase in Periodontal Interleukin-6 Expression across Patients with Neither Diabetes nor Periodontal Disease, Patients with Periodontal Disease Alone, and Patients with Both Diseases," Journal of Periodontal Research, 43(6), 717-22.

Correa, F. O. B., Goncalves, D., Figueredo, C. M. S., Gustafsson, A. \& Orrico, S. R. P. (2008). "The Short-Term Effectiveness of NonSurgical Treatment in Reducing Levels of Interleukin-1beta and Proteases in Gingival Crevicular Fluid from Patients with Type 2 Diabetes Mellitus and Chronic Periodontitis," Journal of Periodontology, 79(11), 2143-50.

Costa, P. P., Trevisan, G. L., Macedo, G. O., Palioto, D. B., Souza, S. L. S., Grisi, M. F. M., Novaes, A. B., Jr. \& Taba, M., Jr. (2010). "Salivary Interleukin-6, Matrix Metalloproteinase-8, and Osteoprotegerin in Patients with Periodontitis and Diabetes," Journal of Periodontology, 81(3), 384-91.

Cutando, A., Gomez-Moreno, G., Villalba, J., Ferrera, M. J., Escames, G. \& AcunaCastroviejo, D. (2003). "Relationship between Salivary Melatonin Levels and Periodontal Status in Diabetic Patients," Journal of Pineal Research, 35(4), 239-44.

Dag, A., Firat, E. T., Arikan, S., Kadiroglu, A. K. \& Kaplan, A. (2009). "The Effect of
Periodontal Therapy on Serum TNF-alpha and HbA1c Levels in Type 2 Diabetic Patients," Australian Dental Journal, 54(1), 17-22.

Engebretson, S., Chertog, R., Nichols, A., Hey-Hadavi, J., Celenti, R. \& Grbic, J. (2007). "Plasma Levels of Tumour Necrosis FactorAlpha in Patients with Chronic Periodontitis and Type 2 Diabetes," Journal of Clinical Periodontology, 34(1), 18-24.

Engebretson, S. P., Vossughi, F., Hey-Hadavi, J., Emingil, G. \& Grbic, J. T. (2006). "The Influence of Diabetes on Gingival Crevicular Fluid Beta-Glucuronidase and Interleukin-8," Journal of Clinical Periodontology, 33(11), 784-90.

Esposito, K., Nappo, F., Marfella, R., Giugliano, G., Giugliano, F., Ciotola, M., Quagliaro, L., Ceriello, A. \& Giugliano, D. (2002). "Inflammatory Cytokine Concentrations are Acutely Increased by Hyperglycemia in Humans: Role of Oxidative Stress," Circulation, 106(16), 2067-72.

Fitzsimmons, T. R., Sanders, A. E., Bartold, P. M. \& Slade, G. D. (2010). "Local and Systemic Biomarkers in Gingival Crevicular Fluid Increase Odds of Periodontitis," Journal of Clinical Periodontology, 37(1), 30-6.

Gemmell, E. \& Seymour, G. J. (1994). "Modulation of Immune Responses to Periodontal Bacteria," Current Opinion in Periodontology, 28-38.

Gimbrone, M. A., Jr., Cybulsky, M. I., Kume, N., Collins, T. \& Resnick, N. (1995). "Vascular Endothelium. An Integrator of Pathophysiological Stimuli in Atherogenesis," Annals of the New York Academy of Sciences, 748, 122-31; discussion 131-2.

Gomes, M. A. B., Rodrigues, F. H., AfonsoCardoso, S. R., Buso, A. M., Silva, A. G., Favoreto, S., Jr. \& Souza, M. A. (2006). "Levels of Immunoglobulin A1 and Messenger RNA for Interferon Gamma and Tumor Necrosis Factor Alpha in Total Saliva from Patients with Diabetes Mellitus Type 2 with Chronic Periodontal Disease," Journal of Periodontal Research, 41(3), 177-83. 
Grossi, S. G. \& Genco, R. J. (1998). "Periodontal Disease and Diabetes Mellitus: A Two-Way Relationship," Annals of Periodontology, 3(1), 51-61.

Hiroshima, Y., Bando, M., Inagaki, Y., Mihara, C., Kataoka, M., Murata, H., Shinohara, Y., Nagata, T. \& Kido, J. (2012). "Resistin in Gingival Crevicular Fluid and Induction of Resistin Release by Porphyromonas Gingivalis Lipopolysaccharide in Human Neutrophils," Journal of Periodontal Research, 47(5), 554-62.

Holloway, A. F., Rao, S. \& Shannon, M. F. (2002). "Regulation of Cytokine Gene Transcription in the Immune System," Molecular Immunology, 38(8), 567-80.

Ioannidou, E., Kao, D., Chang, N., Burleson, J. \& Dongari-Bagtzoglou, A. (2006). "Elevated Serum Interleukin-6 (IL-6) in Solid-Organ Transplant Recipients is Positively Associated with Tissue Destruction and IL6 Gene Expression in the Periodontium," Journal of Periodontology, 77(11), 1871-8.

Javed, F., Al-Askar, M., Samaranayake, L. P. \& Al-Hezaimi, K. (2013a). "Periodontal Disease in Habitual Cigarette Smokers and Nonsmokers with and Without Prediabetes," American Journal of the Medical Sciences, 345(2), 94-8.

Javed, F., Klingspor, L., Sundin, U., Altamash, M., Klinge, B. \& Engstrom, P. E. (2009). "Periodontal Conditions, Oral Candida Albicans and Salivary Proteins in Type 2 Diabetic Subjects with Emphasis on Gender," BMC Oral Health, 9, 12.

Javed, F., Nasstrom, K., Benchimol, D., Altamash, M., Klinge, B. \& Engstrom, P. E. (2007). "Comparison of Periodontal and Socioeconomic Status between Subjects with Type 2 Diabetes Mellitus and NonDiabetic Controls," Journal of Periodontology, 78(11), 2112-9.

Javed, F. \& Romanos, G. E. (2009). "Impact of Diabetes Mellitus and Glycemic Control on the Osseointegration of Dental Implants: A Systematic Literature Review," Journal of Periodontology, 80(11), 1719-30.
Javed, F., Thafeed Alghamdi, A. S., Mikami, T., Mehmood, A., Ahmed, H. B., Samaranayake, L. P. \& Tenenbaum, H. C. (2013b). "Effect of Glycemic Control on Self-Perceived Oral Health, Periodontal Parameters and Alveolar Bone Loss among Patients with Prediabetes," Journal of Periodontology.

Kardesler, L., Buduneli, N., Biyikoglu, B., Cetinkalp, S. \& Kutukculer, N. (2008). "Gingival Crevicular Fluid PGE2, IL-1beta, tPA, PAI-2 Levels in Type 2 Diabetes and Relationship with Periodontal Disease," Clinical Biochemistry, 41(10-11), 863-8.

Kardesler, L., Buduneli, N., Cetinkalp, S. \& Kinane, D. F. (2010). "Adipokines and Inflammatory Mediators after Initial Periodontal Treatment in Patients with Type 2 Diabetes and Chronic Periodontitis," Journal of Periodontology, 81(1), 24-33.

Kinane, D. F. \& Bartold, P. M. (2007). "Clinical Relevance of the Host Responses of Periodontitis," Periodontology 2000, 43, 27893.

Lalla, E., Kaplan, S., Chang, S.- M. J., Roth, G. A., Celenti, R., Hinckley, K., Greenberg, E. \& Papapanou, P. N. (2006). "Periodontal Infection Profiles in Type 1 Diabetes," Journal of Clinical Periodontology, 33(12), 855-62.

Lalla, E., Kaplan, S., Yang, J., Roth, G. A., Papapanou, P. N. \& Greenberg, S. (2007). "Effects of Periodontal Therapy on Serum Creactive Protein, sE-Selectin, and Tumor Necrosis Factor-Alpha Secretion by Peripheral Blood-Derived Macrophages in Diabetes. A Pilot Study," Journal of Periodontal Research, 42(3), 274-82.

Mealey, B. L. \& Ocampo, G. L. (2007). "Diabetes Mellitus and Periodontal Disease," Periodontology 2000, 44, 127-53.

Nareika, A., Maldonado, A., He, L., Game, B. A., Slate, E. H., Sanders, J. J., London, S. D., Lopes-Virella, M. F. \& Huang, Y. (2007). "High Glucose-Boosted Inflammatory Responses to Lipopolysaccharide are Suppressed by Statin," Journal of Periodontal Research, 42(1), 31-8. 
Navarro-Sanchez, A. B., Faria-Almeida, R. \& Bascones-Martinez, A. (2007). "Effect of Non-Surgical Periodontal Therapy on Clinical and Immunological Response and Glycaemic Control in Type 2 Diabetic Patients with Moderate Periodontitis," Journal of Clinical Periodontology, 34(10), 835-43.

Nishimura, F. \& Murayama, Y. (2001). "Periodontal Inflammation and Insulin Resistance--Lessons from Obesity," Journal of Dental Research, 80(8), 1690-4.

Nishimura, F., Taniguchi, A., YamaguchiMorimoto, M., Soga, Y., Iwamoto, Y., Kokeguchi, S., Kuroe, A., Fukushima, M., Nakai, Y. \& Seino, Y. (2006). "Periodontal Infection and Dyslipidemia in Type 2 Diabetics: Association with Increased HMGCoA Reductase Expression," Hormone and Metabolic Research, 38(8), 530-5.

O'Connell, P. A. A., Taba, M., Nomizo, A., Foss Freitas, M. C., Suaid, F. A., Uyemura, S. A., Trevisan, G. L., Novaes, A. B., Souza, S. L., Palioto, D. B. \& Grisi, M. F. (2008). "Effects of Periodontal Therapy on Glycemic Control and Inflammatory Markers," Journal of Periodontology, 79(5), 774-83.

Pradeep, A. R., Raghavendra, N. M., Sharma, A., Patel, S. P., Raju, A., Kathariya, R., Rao, N. S. \& Naik, S. B. (2012). "Association of Serum and Crevicular Visfatin Levels in Periodontal Health and Disease with Type 2 Diabetes Mellitus," Journal of Periodontology, 83(5), 629-34.

Pradeep, A. R., Thorat Manojkumar, S., Garima, G. \& Raju, A. (2010). "Serum Levels of Oncostatin M (a gp 130 Cytokine): An Inflammatory Biomarker in Periodontal Disease," Biomarkers, 15(3), 277-82.

Salvi, G. E., Beck, J. D. \& Offenbacher, S. (1998). "PGE2, IL-1 Beta, and TNF-Alpha Responses in Diabetics as Modifiers of Periodontal Disease Expression," Annals of Periodontology, 3(1), 40-50.

Salvi, G. E., Collins, J. G., Yalda, B., Arnold, R. R., Lang, N. P. \& Offenbacher, S. (1997a). "Monocytic TNF Alpha Secretion Patterns in IDDM Patients with Periodontal Diseases," Journal of Clinical Periodontology, 24(1), 8-
16.

Salvi, G. E., Franco, L. M., Braun, T. M., Lee, A., Rutger Persson, G., Lang, N. P. \& Giannobile, W. V. (2010). "Pro-inflammatory Biomarkers during Experimental Gingivitis in Patients with Type 1 Diabetes Mellitus: A Proof-of-Concept Study," Journal of Clinical Periodontology, 37(1), 9-16.

Salvi, G. E., Yalda, B., Collins, J. G., Jones, B. H., Smith, F. W., Arnold, R. R. \& Offenbacher, S. (1997b). "Inflammatory Mediator Response as a Potential Risk Marker for Periodontal Diseases in Insulin-Dependent Diabetes Mellitus Patients," Journal of Periodontology, 68(2), 127-35.

Suh, K.- I., Kim, Y.- K. \& Kho, H.- S. (2009). "Salivary Levels of IL-1beta, IL-6, IL-8, and TNF-Alpha in Patients with Burning Mouth Syndrome," Archives of Oral Biology, 54(9), 797-802. 